\title{
CUBIC EQUATIONS FOR THE HYPERELLIPTIC LOCUS *
}

\author{
SAMUEL GRUSHEVSKY ${ }^{\dagger}$
}

\begin{abstract}
We prove a conjecture from [BK2] that the multi-dimensional vector addition formula for Baker-Akhiezer functions obtained there characterizes Jacobians among principally polarized abelian varieties. We also show that this addition formula is equivalent to Gunning's multisecant formula for the Kummer variety obtained in [Gu2].

We then use this addition formula to obtain cubic relations among theta functions that characterize the locus of hyperelliptic Jacobians among irreducible abelian varieties. In genus 3 our equations are equivalent to the vanishing of one theta-null, and thus are classical (see $[\mathrm{M}],[\mathrm{P}]$ ), but already for genus 4 they appear to be new.
\end{abstract}

1. Definitions and notations. We work over $\mathbb{C}$, and fix the dimension/genus $g>1$. Let $\mathcal{H}_{g}$ be the Siegel upper half-space - the set of all $g \times g$ period matrices $\tau$, i.e. symmetric complex $g \times g$ matrices with positive definite imaginary part. Each such $\tau$ corresponds to an abelian variety $X_{\tau}:=\mathbb{C}^{g} / \tau \mathbb{Z}^{g}+\mathbb{Z}^{g}$, and the moduli space $\mathcal{A}_{g}$ of principally polarized abelian varieties (ppavs) is then the quotient of $\mathcal{H}_{g}$ by a certain action of the symplectic group $\operatorname{Sp}(2 g, \mathbb{Z})$.

A ppav is called irreducible if it is not isomorphic to a product of two lowerdimensional ppavs (with polarization). For convenience we denote by $\mathcal{A}_{g}^{i r r}$ the moduli space of irreducible ppavs of genus $g$. When in the following we say "abelian variety", we actually mean a ppav.

Denoting $\mathbf{e}(x):=\exp (\pi i x)$, for a period matrix $\tau$ and a vector $z \in \mathbb{C}^{g}$ we define the theta function with characteristics $\varepsilon, \delta \in(\mathbb{Z} / 2 \mathbb{Z})^{g}$, thought of as vectors consisting of zeros and ones, to be

$$
\theta\left[\begin{array}{l}
\varepsilon \\
\delta
\end{array}\right](\tau, z):=\sum_{m \in \mathbb{Z}^{g}} \mathbf{e}\left[\left(m+\frac{\varepsilon}{2}, \tau\left(m+\frac{\varepsilon}{2}\right)\right)+2\left(m+\frac{\varepsilon}{2}, z+\frac{\delta}{2}\right)\right]
$$

where $(\cdot, \cdot)$ denotes the scalar product. A theta function with characteristics is even or odd as a function of $z$ depending on whether the scalar product $(\varepsilon, \delta)$ is even or odd.

We denote by $\theta(\tau, z):=\theta\left[\begin{array}{l}0 \\ 0\end{array}\right](\tau, z)$ the classical Riemann's theta function. Theta functions with characteristics are, up to a constant factor, just the values of Riemann's theta function of a shifted argument:

$$
\begin{aligned}
& \theta\left(\tau, z+\frac{\tau \varepsilon+\delta}{2}\right)=\sum_{m \in \mathbb{Z}^{g}} \mathbf{e}\left[(m, \tau m)+2\left(m, z+\frac{\tau \varepsilon+\delta}{2}\right)\right] \\
& \quad=\sum_{m \in \mathbb{Z}^{g}} \mathbf{e}\left[\left(m+\frac{\varepsilon}{2}, \tau\left(m+\frac{\varepsilon}{2}\right)\right)+2\left(m+\frac{\varepsilon}{2}, z+\frac{\delta}{2}\right)-\left(\frac{\varepsilon}{2}, \tau \frac{\varepsilon}{2}\right)-\left(\varepsilon, z+\frac{\delta}{2}\right)\right] \\
& \quad=(-1)^{(\varepsilon, \delta)} \mathbf{e}\left[-\frac{1}{4}(\varepsilon, \tau \varepsilon)-(\varepsilon, z)\right] \theta\left[\begin{array}{l}
\varepsilon \\
\delta
\end{array}\right](\tau, z) .
\end{aligned}
$$

Thus instead of thinking of a characteristic $\left[\begin{array}{l}\varepsilon \\ \delta\end{array}\right]$ as two integer vectors it sometimes is better to think of it as the point $\frac{\tau \varepsilon+\delta}{2}$ of order two on the abelian variety $X_{\tau}$.

\footnotetext{
*Received May 5, 2003; accepted for publication July 8, 2003.

$\dagger^{\dagger}$ Mathematics Department, Princeton University, Fine Hall, Washington Road, Princeton, NJ 08544, USA (sam@math.princeton.edu). Partially supported by NSF Mathematical Sciences Postdoctoral Research Fellowship.
} 
We further define theta functions of the second order to be

$$
\Theta[\varepsilon](\tau, z):=\theta\left[\begin{array}{l}
\varepsilon \\
0
\end{array}\right](2 \tau, 2 z)
$$

For a fixed $\tau$ the theta functions, as functions of the variable $z$, are sections of certain bundles on the abelian variety $X_{\tau}$, which is to say that if the variable $z$ is translated by a vector of the lattice $\tau \mathbb{Z}^{g}+\mathbb{Z}^{g}$, theta functions multiply by a certain number. In fact it is known that all theta functions of the second order are sections of the same bundle, denoted $2 \Theta$, and transform as follows:

$$
\Theta[\varepsilon]\left(\tau, z+e_{j}+\tau e_{k}\right)=\mathbf{e}\left(-2\left(e_{k}, \tau e_{k}\right)-4\left(e_{k}, z\right)\right) \Theta[\varepsilon](\tau, z),
$$

where we denote by $e_{k}$ the basis vector for the $k^{\prime}$ th direction in $\mathbb{C}^{g}$.

Theta functions of the second order form a basis for the sections of $2 \Theta$ over $X_{\tau}$. The square of any theta function with characteristics is also a section of $2 \Theta$, and thus is expressible as a linear combination of theta functions of the second order. In fact a slightly more general formula, Riemann's bilinear addition theorem, holds:

$$
\theta\left[\begin{array}{l}
\varepsilon \\
\delta
\end{array}\right](z) \theta\left[\begin{array}{l}
\varepsilon \\
\delta
\end{array}\right](w)=\sum_{\sigma \in(\mathbb{Z} / 2 \mathbb{Z})^{g}}(-1)^{(\delta, \sigma)} \Theta[\sigma+\varepsilon]\left(\frac{z+w}{2}\right) \Theta[\sigma]\left(\frac{z-w}{2}\right) .
$$

For a fixed $\tau$ the map $z \rightarrow\{\Theta[\varepsilon](\tau, z)\}_{\text {all } \varepsilon}$ defines the Kummer embedding $K$ : $X_{\tau} / \pm 1 \rightarrow \mathbb{P}^{2^{g}-1}$. This map is well-defined since all theta functions of the second order are sections of the same line bundle, and are even in $z$.

The values of theta functions at $z=0$ are called the associated theta constants. Theta constants of the second order are modular forms of weight one half with respect to a certain finite index normal subgroup $\Gamma(2,4) \subset \operatorname{Sp}(2 g, \mathbb{Z})$, which is to say that if we act upon $\tau$ by some $\gamma \in \Gamma(2,4)$, then $\Theta[\varepsilon](\gamma \tau)=k(\gamma, \tau) \Theta[\varepsilon](\tau)$, where $k$ is some multiplier depending on $\gamma$ and $\tau$, but independent of $\varepsilon$. Thus letting $\mathcal{A}_{g}(2,4):=\mathcal{H}_{g} / \Gamma(2,4)$, we see that theta constants of the second order define a map $T h: \mathcal{A}_{g}(2,4) \rightarrow \mathbb{P}^{2^{g}-1}$, which is known to be generically injective for all genera, and injective for $g \leq 3$. The level moduli space $\mathcal{A}_{g}(2,4)$ is a finite cover of $\mathcal{A}_{g}$.

Let us denote by $\mathcal{J}_{g} \subset \mathcal{A}_{g}$ the locus of Jacobians of Riemann surfaces of genus $g$, and by $\mathcal{I}_{g} \subset \mathcal{J}_{g}$ - the locus of Jacobians of hyperelliptic Riemann surfaces. The question of characterizing $\mathcal{J}_{g}$ within $\mathcal{A}_{g}$ is called the Schottky problem, and that of characterizing $\mathcal{I}_{g}$ - the Schottky problem for the hyperelliptics. More precisely, one takes the preimages $\mathcal{J}_{g}(2,4)$ and $\mathcal{I}_{g}(2,4)$ of $\mathcal{J}_{g}$ and $\mathcal{I}_{g}$, respectively, under the covering map $\pi: \mathcal{A}_{g}(2,4) \rightarrow \mathcal{A}_{g}$, and asks to describe $\operatorname{Th}\left(\mathcal{J}_{g}(2,4)\right)$ and $\operatorname{Th}\left(\mathcal{I}_{g}(2,4)\right)$ inside $T h\left(\mathcal{A}_{g}(2,4)\right)$. The question of describing $T h\left(\mathcal{A}_{g}(2,4)\right) \subset \mathbb{P}^{2^{g}-1}$, i.e. determining all the relations in the subring of the ring of modular forms generated by theta constants is also of interest, but we will not discuss it here. Notice that $\mathcal{J}_{g}$ and $\mathcal{I}_{g}$ are irreducible, while $\mathcal{J}_{g}(2,4)$ and $\mathcal{I}_{g}(2,4)$ have many irreducible components. We refer the reader to [I] for more details on theta functions, and to [Gr] for more details on the Schottky problem.

We will always think of a curve $C$ embedded in its Jacobian by the Abel-Jacobi map $A: C \rightarrow J(C)$ with some choice of the basis for the space of holomorphic differentials and of the starting point $P$ made. This choice will be made explicitly when necessary. To avoid technical difficulties in the following sections, it will often be easier to work with the universal cover $\tilde{C}$ of a curve $C$ and the universal cover $\mathbb{C}^{g}$ of the abelian variety $X_{\tau}$, and later take the automorphy properties of theta functions 
into account. The abelian variety will be fixed throughout, and thus we will often omit $\tau$ from the notations for theta functions and constants.

In this work we first prove a conjecture stated in [BK2] that the validity of a certain $g$-dimensional addition formula developed there and in [BK1] characterizes Jacobians, and then proceed to obtain from this some explicit identities for theta functions of hyperelliptic curves. In [M], theorem 9.1 and references therein Mumford showed that the hyperelliptic locus is characterized by a certain set of vanishing and non-vanishing conditions for theta constants with characteristics (the idea goes back at least to Thomae, see $[\mathrm{T}])$. In $[\mathrm{P}]$ Poor showed that on $\mathcal{A}_{g}^{\text {irr }}$ Mumford's vanishing conditions (without the non-vanishing) define precisely the hyperelliptic locus, i.e. that there are no extra components. However, it is still not known how to obtain an ideal-theoretic description of the closure of $T h\left(\mathcal{I}_{g}\right)$ inside $T h\left(\mathcal{A}_{g}\right)$. It is known that if the vanishing holds and we also have some vanishing instead of non-vanishing, the abelian variety must be reducible, but then it does not necessarily have to be a limit of hyperelliptic Jacobians. Thus it would be interesting to study our equations on the reducible locus. It would also be very interesting to compare Mumford's equations to ours, but we have not been able to achieve this yet.

We would also like to refer to a recent work [SM] for a further discussion of these issues as well as a description of components of $\mathcal{I}_{g}^{2,4}$ as locally complete intersections.

2. Addition formula and multisecants. From [BK2] we know the following addition formula (called "formula" to distinguish it from Riemann's addition "theorem"):

FACT 1 ([BK2], theorem 1). Let $P:=A_{0}, A_{1}, \ldots, A_{g}, Q:=A_{g+1} \in \tilde{C} \subset \mathbb{C}^{g}$, and denote by $R:=K-\sum_{i=1}^{g} A_{i}$ the vector of Riemann constants shifted by $-\sum A_{i}$. Then for all $x, y \in \mathbb{C}^{g}$ the following identity is satisfied:

$$
\begin{gathered}
\frac{\theta(Q+x+y+R) \theta(P+R)}{\theta(Q+R) \theta(P+x+y+R)}= \\
=\frac{\theta(Q+x+R) \theta(P+R)}{\theta(Q+R) \theta(P+x+R)} \cdot \frac{\theta(Q+y+R) \theta(P+R)}{\theta(Q+R) \theta(P+y+R)} \\
-\sum_{k=1}^{g} \frac{\theta\left(Q+R+A_{k}-P\right) \theta\left(Q+R-A_{k}+P+x+y\right)}{\theta^{2}(Q+R) \theta(P+x+y+R) \theta\left(2 A_{k}-P+R\right)} \\
. \frac{\theta\left(R+A_{k}+x\right) \theta(P+R)}{\theta(P+x+R)} \cdot \frac{\theta\left(R+A_{k}+y\right) \theta(P+R)}{\theta(P+y+R)} .
\end{gathered}
$$

Though this formula may look formidable, it is very explicit and is written entirely in terms of theta functions. In the following, we take the Abel-Jacobi map to start at $P$, so that $P=0 \in \mathbb{C}^{g}$. Upon cancellations and multiplication by the common denominators, the above formula becomes simply

$$
\theta(Q+x+y+R) \theta(Q+R) \theta(x+R) \theta(y+R)=
$$




$$
\begin{gathered}
=\theta(x+y+R) \theta(R) \theta(Q+x+R) \theta(Q+y+R) \\
-\sum_{k=1}^{g} \frac{\theta(R) \theta\left(Q+A_{k}+R\right) \theta\left(Q-A_{k}+x+y+R\right) \theta\left(A_{k}+x+R\right) \theta\left(A_{k}+y+R\right)}{\theta\left(2 A_{k}+R\right)}
\end{gathered}
$$

To see that this is in fact equivalent to Gunning's general multisecant formula from [Gu2] (see Poor's work [P] for an in-depth discussion) we use Riemann's bilinear addition theorem for the last two factors of each term. Denoting $z:=\frac{x+y}{2}$ and $w:=\frac{x-y}{2}$, notice that the half-difference is always simply $\frac{x-y}{2}$, so we will have a common factor of $\Theta[\sigma](w)$, and the resulting equation will be

$$
\begin{aligned}
0= & \sum_{\sigma}[\theta(Q+2 z+R) \theta(Q+R) \Theta[\sigma](z+R)-\theta(2 z+R) \theta(R) \Theta[\sigma](Q+z+R) \\
& \left.+\sum_{k=1}^{g} \frac{\theta(R) \theta\left(Q+A_{k}+R\right) \theta\left(Q-A_{k}+2 z+R\right) \Theta[\sigma]\left(A_{k}+z+R\right)}{\theta\left(2 A_{k}+R\right)}\right] \Theta[\sigma](w)
\end{aligned}
$$

In the above the coefficient in the square brackets does not depend on $w$, so we have an equation $\sum_{\sigma} b_{\sigma} \Theta[\sigma](w)=0 \forall w$, where the coefficients $b_{\sigma}$ do not depend on $w$. Since theta functions of the second order are a basis for sections of $2 \Theta$ and thus linearly independent, it means that all coefficients $b_{\sigma}$ must be zero. Then since $b_{\sigma}$ actually are some functions of $z$ multiplied by $\Theta[\sigma]\left(A_{i}+z+R\right)$ ( $i$ here ranges from 0 to $g+1$, i.e. includes $P$ and $Q$ ), we have

$$
\forall \sigma, \forall z \quad \sum_{i=0}^{g+1} c_{i}\left(z, A_{i}\right) \Theta[\sigma]\left(A_{i}+z+R\right)=0
$$

for appropriate $c_{i}$ 's.

Since $R$ does not depend on $z$, in the above we can shift $z$ by $R$ and redefine the functions $c_{i}$ to see that the addition formula of [BK2] implies the existence of holomorphic functions $c_{i}$, never all vanishing simultaneously, such that for some fixed $A$ 's lying on the image $A(C) \subset J(C)$ we have

$$
\sum_{i=0}^{g+1} c_{i}(z) K\left(A_{i}+z\right)=0 \quad \forall z \in \mathbb{C}^{g},
$$

which is equivalent to saying that the $g+2$ points $K\left(A_{i}+z\right)$ lie on a $g$-plane in $\mathbb{P}^{2^{g}}-1$. In fact Gunning proves a more general theorem:

FACT 2 ([Gu2], theorem 2). For any curve $C$ of genus $g$, for any $1 \leq m \leq g$ and for any points $x_{1}, \ldots, x_{m}, A_{0}, \ldots, A_{m+1} \in A(C)$ the $m+2$ points $K\left(A_{i}+\sum x-\sum A\right)$ are collinear, i.e. lie on the intersection of the Kummer variety of $C$ with an $m$-plane in the projective space $\mathbb{P}^{2^{g}-1}$.

In particular since the $g^{\prime}$ th symmetric power of the curve is its Jacobian, $S^{g}(A(C))=J(C)$, the case $m=g$ of this theorem is formula (5), while the case $m=1$ is the case of a family of trisecant lines. It is shown in [Gu1] that the existence of a family of trisecants characterizes Jacobians among irreducible ppavs. 
3. Characterizing Jacobians by families of multisecants. We will now show that Buchstaber-Krichever's addition formula and Gunning's multisecant formula also characterize Jacobians.

THEOREM 3. Let $X$ be an irreducible principally polarized abelian variety of dimension $g$, and let $A_{0}, \ldots, A_{g+1}$ be distinct points of $X$. Suppose that $\forall z \in X$ the $g+2$ points $K\left(A_{i}+z\right)$ are linearly dependent. Then $X$ is the Jacobian of some curve $C$, and $A_{i} \in A(C)$.

Proof. Assume $g \geq 4$, otherwise the theorem is trivial. Working in the spirit of Gunning's work [Gu1], we reduce the theorem to the case of the trisecant.

Indeed, formula (5) is a large number of linear conditions on $c_{i}(z)$. If we can pick characteristics $\varepsilon_{0} \ldots \varepsilon_{g}$ so that the matrix

$$
\left(\begin{array}{ccc}
\Theta\left[\varepsilon_{0}\right]\left(A_{0}+z\right) & \ldots & \Theta\left[\varepsilon_{0}\right]\left(A_{g+1}+z\right) \\
\vdots & \ddots & \vdots \\
\Theta\left[\varepsilon_{g}\right]\left(A_{0}+z\right) & \ldots & \Theta\left[\varepsilon_{g}\right]\left(A_{g+1}+z\right)
\end{array}\right)
$$

has rank $g+1$, then each $c_{i}(z)$ is the determinant of this matrix with $i$ 's column deleted, multiplied by $(-1)^{i}$.

LemMA 4. The functions $c_{i}(z)$ defined as the appropriate determinants above can be continued to all of $\mathbb{C}^{g}$ to define a holomorphic map $\vec{c}: \mathbb{C}^{g} \rightarrow \mathbb{P}^{g+1}$ such that (5) is satisfied.

Proof. If a choice of $\varepsilon$ 's as above is impossible, it means that the $g+2$ vectors $K\left(A_{i}+z\right)$ actually lie on a (projective) $(g-1)$-plane instead of a $g$-plane. Suppose in fact their linear span is generated by $K\left(A_{i_{1}}+z\right), \ldots, K\left(A_{i_{k}}+z\right)$. Here $k$ must be at least 2 and at most $g$. Indeed if $k$ is 1 , then since $K$ is injective on $X / \pm 1$, it would mean that all $A_{j}+z$ are equal to $\pm\left(A_{i_{1}}+z\right)$ and in particular at least two of $A_{j}$ 's would coincide. Now assume $n \notin\left\{i_{1} \ldots i_{k}\right\}$ and $A_{n}+z$ is not of order two, and vary the point $A_{n}$ by adding to it a small vector $v$. Since by a theorem of Wirtinger (see [Wi]) for any $y \in X_{\tau}$ not of order two the $g+1$ vectors $K(y)$ and $\frac{\partial}{\partial z_{j}} K(y)$ are linearly independent, it means that for some $j$ the vector $\frac{\partial}{\partial z_{j}} K\left(A_{n}+z\right)$ does not lie in the linear span of $K\left(A_{i_{1}}+z\right), \ldots, K\left(A_{i_{k}}+z\right)$, and thus by infinitesimally varying $A_{n}$ in some direction we can make $K\left(A_{n}+z\right)$, which was a linear combination of $K\left(A_{i_{j}}+z\right)$, linearly independent with these $k$ vectors. Varying if necessary a few more of the $A$ 's we can then increase the rank of the $(g+2) \times 2^{g}$ matrix $K\left(A_{0}+x\right), \ldots, K\left(A_{g+1}+x\right)$ to $g+1$, and then all the determinants needed to compute $c_{i}$ 's will be non-zero. We then compute these $c_{i}$ 's after a small perturbation of $A$ 's as above and then take the limit of the point $\vec{c} \in \mathbb{P}^{g+1}$ as the $A$ 's return to their original positions.

If in fact in the above we had $A_{n}+x$ of order two no matter what index $n$ we pick (in particular all points $A_{i}$ differ by vectors of order two, and this is the case which is needed for studying the hyperelliptic Jacobians), then we note that the functions $c_{i}$ can be defined uniquely for all $y$ 's near $z$, as then $A_{n}+y$ is not of order two. Then since $c$ 's are given as polynomials in thetas, their ratios cannot have essential singularities and thus can be continued to the point $z$.

REMARK 5. It would be nice to have a map $\vec{c}: X \rightarrow \mathbb{P}^{g+1}$ instead of a map from $\mathbb{C}^{g}$, but unfortunately this is usually impossible. Indeed, the multipliers for different functions $\theta\left(A_{i}+z\right)$ from formula (2) are different (unless $A_{i}$ differ only by half-integer 
vectors), i.e. they are sections of different bundles on $X$, and thus the map from $X$ to $\mathbb{P}^{g}$ is not well-defined.

We will show that $p:=(1:-1: 0: \ldots: 0)=\vec{c}(y)$ for some $y \in \mathbb{C}^{g}$ and that the differential $\mathrm{d} \vec{c}: T_{y} \mathbb{C}^{g} \rightarrow T_{p} \mathbb{P}^{g+1}$ is of maximal rank. This will then imply that locally near $p$ the image $\vec{c}\left(\mathbb{C}^{g}\right)$ is of dimension $g$ and thus locally the preimage of some coordinate plane $\mathbb{P}^{2} \subset \mathbb{P}^{g+1}$ containing $p$ is at least one-dimensional. Then we have a one-dimensional family of trisecants of the Kummer variety, and by Welters' [We] infinitesimal version of Gunning's trisecant criterion from [Gu1], $X$ is a Jacobian of some curve $C$ with the points $A_{0}, A_{1}, A_{2}$ lying on $A(C)$. Changing the point $p$ by renumbering coordinates we see that all $A_{i}$ lie on $A(C)$.

Indeed let $y:=-\frac{A_{0}+A_{1}}{2}$ as a point of $\mathbb{C}^{g}$. Then $A_{0}+y=-\left(A_{1}+y\right)\left(\right.$ in $\left.\mathbb{C}^{g}\right)$ and, since theta functions are even, $K\left(A_{0}+y\right)=K\left(A_{1}+y\right)$ (as points of $\mathbb{C}^{g}$, nonprojectivized), which means that $\vec{c}(y)=p$ (if we compute the determinants above, all the $c_{i}(y)$ with $i \geq 2$ will vanish as the matrix will have two identical columns, and the determinants for $c_{0}(y)$ and $c_{1}(y)$ will be the same, but taken with opposite signs). Now suppose that the rank of $\left.\mathrm{d} \vec{c}\right|_{y}$ is not maximal, i.e. that there is some vector $v \in \mathbb{C}^{g}$ such that for all $i$ we have $\left.\frac{\partial}{\partial v} c_{i}(z)\right|_{y}=\lambda c_{i}(p)$ for some constant $\lambda$ independent of $i$ - this means that the derivative $\frac{\partial}{\partial v}$ of the projective point $\vec{c}$ is zero.

Let us take the derivative $\frac{\partial}{\partial v}$ of $(5)$ at $y$ :

$$
\begin{gathered}
\sum_{i=0}^{g+1} \frac{\partial c_{i}(z)}{\partial v} K\left(A_{i}+z\right)+\left.c_{i}(z) \frac{\partial K}{\partial v}\left(A_{i}+z\right)\right|_{y} \\
=\lambda c_{0}(y)\left(K\left(A_{0}+y\right)-K\left(A_{1}+y\right)\right)+c_{0}(y)\left(\frac{\partial K}{\partial v}\left(A_{0}+y\right)-\frac{\partial K}{\partial v}\left(A_{1}+y\right)\right) \\
=2 c_{0}(y) \frac{\partial K}{\partial v}\left(A_{0}+y\right)=0,
\end{gathered}
$$

because theta functions are even and their derivatives are odd. But this then implies that $\frac{\partial K}{\partial v}\left(A_{0}+y\right)=0$, which is impossible by Wirtinger's theorem unless $A_{0}+y=$ $\frac{A_{0}-A_{1}}{2}$ is a point of order two in $X$. If this is the case, though, it would mean that $A_{0}-A_{1}=0 \in X$, which contradicts the assumption that all $A_{i}$ are distinct. Thus we have arrived at a contradiction and showed that the differential $\mathrm{d} \vec{c}: T_{y} \mathbb{C}^{g} \rightarrow T_{P} \mathbb{P}^{g+1}$ is injective.

With more work it can also be shown that Gunning's addition formula for all other values of $m$ also serves to characterize Jacobians:

Proposition 6. Let $X$ be an irreducible principally polarized abelian variety of dimension $g$, and let $A_{0}, \ldots, A_{m+1}$ be different points of $X$. Suppose that the $m+2$ points $K\left(A_{i}+z\right)$ are linearly dependent for any $z \in M$, where the set $M \subset X$ is at least $m$-dimensional at the point $y=-\frac{A_{i}+A_{j}}{2}$ for some $i$ and $j$ (i.e. has a non-degenerate $m$-jet at $y)$. Then $X$ is the Jacobian of some curve.

Proof. The proof is analogous to the one above. We take the largest $M$ possible, and then the lemma still works to define a map $\vec{c}_{m}: M \rightarrow \mathbb{P}^{m+1}$. Then we want to show that $\mathrm{d} \vec{c}$ is injective on $T_{y} \tilde{M}$. But since $\mathrm{d} \vec{c}$ is non-degenerate on $T_{y} \mathbb{C}^{g}$, it is also non-degenerate on a subspace, and we are done.

REMARK 7. By imitating the Gunning's proof of the trisecant theorem in [Gu1] it seems to be also possible to show that if $M$ is $m$-dimensional at some point, not necessarily $-\frac{A_{i}+A_{j}}{2}$, then $X$ is a Jacobian. However, as we do not need this result in 
what follows, and the proof would be technically rather complicated, we will not give it.

In [Gu2] Gunning shows that for a Jacobian $X$ with fixed $A$ 's the coefficients $c_{i}$ in (5) are unique up to scaling and expressible in terms of the Klein-Gunning prime form. The Klein-Gunning prime form expression is rather hard to deal with (see $[\mathrm{P}]$ for a detailed discussion and computations); however, the expression for $c_{i}$ obtained in formula (4) seems more amenable.

Equation (4) includes, however, both theta functions of the second order and the classical Riemann's theta function with zero characteristic. Let us use the addition theorem once again for the last two factors of the type $\theta(\ldots) \theta(\ldots)$ in each term. We then get for all $\sigma$

$$
\begin{gathered}
\sum_{\varepsilon} \Theta[\varepsilon](Q+z+R) \Theta[\varepsilon](z) \Theta[\sigma](z+R)-\Theta[\varepsilon](z+R) \Theta[\varepsilon](z) \Theta[\sigma](Q+z+R) \\
+\sum_{k, \varepsilon} \frac{\theta(R)}{\theta\left(2 A_{k}+R\right)} \Theta[\varepsilon](Q+z+R) \Theta[\varepsilon]\left(z-A_{k}\right) \Theta[\sigma]\left(A_{k}+z+R\right)=0 .
\end{gathered}
$$

Since all the transformations that we have done so far are equivalencies, we do not lose any information. Moreover, since by Gunning's results in [Gu2] the coefficients $c_{i}$ in (5) are unique, they must be exactly the ones given by formula (6), and thus formula (6) is satisfied if and only if the collinearity condition (5) is satisfied.

Proposition 8. With the same assumptions and notations as above, identity (6) characterizes Jacobians among all irreducible abelian varieties.

4. Addition formula for the hyperelliptic case. Formula (6) we obtained is in terms of theta functions evaluated at different points. In the classical approach to the Schottky problem (see $[\mathrm{S}]$ for the origins and $[\mathrm{F}],[\mathrm{Gr}]$ for a review) one wants to characterize the Jacobian locus by some algebraic relations among theta constants. Thus it would be nice if for some special values of $z, P, Q$ and $A$ 's the addition formula yielded such equations.

However, from the transformation rule (2) for theta functions it is easy to see that if a vector $v$ is not a point of order two, then $\Theta[\varepsilon](z+v)$ is not a section of the bundle $2 \Theta$ and thus cannot be expressed as a linear combination of theta functions of the second order. Thus we only have a reasonable hope of getting from (6) some equations for theta constants if we are so lucky that all the "shifts" of $z$ that appear there are points of order two. In particular, this means that the points $A_{i}+z+R$ must be of order two for all $i$.

Now suppose that indeed both $A_{i}+z+R$ and $A_{j}+z+R$ for $i \neq j$ are points of order two on $J(C)$. Then their difference, $A_{i}-A_{j}$, is also of order two, so we have $2 A_{i}-2 A_{j}=0 \in J(C)$. By Abel's theorem this then means that there is a function $f$ on $C$ whose divisor is equal to $2 A_{i}-2 A_{j}$, i.e. with a double pole at $A_{j}$ and holomorphic on $C-\left\{A_{j}\right\}$. Since the existence of such a function characterizes hyperelliptic curves, it means that $C$ then has to be hyperelliptic. For the hyperelliptic curves it is known (see, for example, $[\mathrm{M}]$ ) that if we take $P=0$ to be the image of one of the Weierstrass points, then the other $2 g+1$ Weierstrass points will also map to points of order two on the Jacobian.

Thus let us assume that all $A$ 's and $Q$ in formula 6 are chosen to be points of order two, i.e. that we are dealing with a hyperelliptic curve, and let us rewrite the addition formula in this case. Denote $Q=\frac{\tau \alpha_{0}+\beta_{0}}{2}, A_{k}=\frac{\tau \alpha_{k}+\beta_{k}}{2}$ and $R=\frac{\tau \alpha+\beta}{2}-$ in fact $R$ is expressible in terms of $A$ 's and Riemann constants, but we will deal with this later. 
Now we rewrite formula (6) for these $A$ 's and $Q$. In doing this, we need to be extra careful to remember that we are actually working on $\tilde{C}$ and $\mathbb{C}^{g}$, as not to omit any important automorphy factors. Indeed, from the automorphy properties of $\theta$ it follows that

$$
\frac{\theta(R)}{\theta\left(2 A_{k}+R\right)}=\frac{\theta\left(\frac{\tau \alpha+\beta}{2}\right)}{\theta\left(\tau \alpha_{k}+\beta_{k}+\frac{\tau \alpha+\beta}{2}\right)}=\mathbf{e}\left[\left(\alpha_{k}, \tau \alpha_{k}\right)+\left(\alpha, \tau \alpha_{k}\right)\right] .
$$

Also for any integers $a$ and $b$ it follows from (1) and (2) that

$$
\begin{aligned}
\Theta[\delta] & \left(\tau, z+\frac{\tau a+b}{2}\right)=\theta\left[\begin{array}{l}
\delta \\
0
\end{array}\right](2 \tau, 2 z+\tau a+b) \\
= & \theta(2 \tau, 2 z+\tau \delta+\tau a) \mathbf{e}\left[\frac{1}{2}(\delta, \tau \delta)+2(\delta, z)+(\delta, \tau a)+(\delta, b)\right] \\
= & \theta\left[\begin{array}{c}
\delta+a \\
0
\end{array}\right](2 \tau, 2 z) \mathbf{e}\left[-\frac{1}{2}(\delta+a, \tau(\delta+a))-2(\delta+a, z)\right] \\
& \cdot \mathbf{e}\left[\frac{1}{2}(\delta, \tau \delta)+2(\delta, z)+(\delta, \tau a)+(\delta, b)\right] \\
= & (-1)^{(\delta, b)} \mathbf{e}\left[-\frac{1}{2}(a, \tau a)-2(a, z)\right] \Theta[\delta+a](\tau, z)
\end{aligned}
$$

When we substitute this into (6) notice that as functions of $z$ all terms are actually sections of the same bundle, $6 \Theta$, as each is cubic in theta functions of the second order. Thus the $\mathbf{e}[(*, z)]$ factors must cancel everywhere. Also evaluating at $z=0$ and noticing that all terms are modular forms in $\tau$ with respect to $\Gamma(2,4)$ of the same weight, we expect the factors $\mathbf{e}[(*, \tau *)]$ to cancel as well. A trivial but tedious computation confirms this, and we arrive at

Proposition 9. An irreducible abelian variety $X_{\tau}$ with some points $P=$ $0, Q, A_{1}, \ldots, A_{g}$ with $A_{i}=\frac{\tau \alpha_{k}+\beta_{k}}{2} \in \mathbb{C}^{g}$ is the Jacobian of a hyperelliptic curve $C$, and $P, Q, A_{i} \in A(\tilde{C}) \subset \mathbb{C}^{g}$ if and only if the following is satisfied for all $\sigma \in(\mathbb{Z} / 2 \mathbb{Z})^{g}$ and for all $z \in \mathbb{C}^{g}$ :

$$
\begin{aligned}
& \sum_{\varepsilon}(-1)^{\left(\varepsilon, \beta+\beta_{0}\right)} \Theta\left[\varepsilon+\alpha+\alpha_{0}\right](z) \Theta[\varepsilon](z) \Theta[\sigma+\alpha](z) \\
= & \sum_{e, k}(-1)^{\left(\varepsilon, \beta+\beta_{0}+\beta_{k}\right)+\left(\sigma, \beta_{k}\right)} \Theta\left[\varepsilon+\alpha+\alpha_{0}\right](z) \Theta\left[\varepsilon+\alpha_{k}\right](z) \Theta\left[\sigma+\alpha+\alpha_{k}\right](z) \\
+ & \sum_{\varepsilon}(-1)^{(\varepsilon, \beta)+\left(\sigma, \beta_{0}\right)} \Theta[\varepsilon+\alpha](z) \Theta[\varepsilon](z) \Theta\left[\sigma+\alpha+\alpha_{0}\right](z) .
\end{aligned}
$$

5. Cubic equations for the hyperelliptic locus. To make formula (8) entirely explicit, we now need to pick some specific way to map a hyperelliptic curve into its Jacobian, and pick some $g+2$ Weierstrass points on it in a certain way. This is indeed a very classical construction.

Let us think of a hyperelliptic curve sitting on a skewer that intersects it in precisely the $2 g+2$ Weierstrass points. Label them $p_{1}, \ldots, p_{2 g+2}$ going from left to right along the skewer. Then pick for the basis of the cycles $a_{i}$ to be the loop around the $i$ 'th handle, passing through points $p_{2 i-1}$ and $p_{2 i}$, and $b_{i}$ to be the loop around the $i$ 'th hole, passing through $p_{2 i}$ and $p_{2 i+1}$. Then thinking of the skewer as being the $x$ axis and the whole picture being that of $y^{2}=\prod\left(x-p_{i}\right)$, we can compute the images 
of $p_{i}$ in the Jacobian. Indeed, let us use $p_{1}$ as the starting point, so that $A\left(p_{1}\right)=0$. Then we see that $A\left(p_{2}\right)=\frac{e_{1}}{2}, A\left(p_{3}\right)=\frac{\tau e_{1}+e_{1}}{2}, A\left(p_{4}\right)=\frac{\tau e_{1}+e_{1}}{2}+\frac{e_{1}+e_{2}}{2}=\frac{\tau e_{1}+e_{2}}{2}+e_{1}$, $A\left(p_{5}\right)=\frac{\tau\left(e_{1}+e_{2}\right)+e_{2}}{2}+e_{1}$, and in general we have $A\left(p_{2 i}\right)=\frac{\tau s_{i-1}+e_{i}}{2}+s_{i-1}$ and $A\left(p_{2 i+1}\right)=\frac{\tau s_{i}+e_{i}}{2}+s_{i-1}$ for $1 \leq i \leq g$, while $A\left(p_{2 g+2}\right)=\frac{\tau s_{g}}{2}$ (where for convenience we have denoted $s_{k}:=\sum_{i=1}^{k} e_{i}$.

For our purposes let us choose $P:=0=A\left(p_{1}\right), Q:=A\left(p_{2}\right)$ and $A_{k}:=A\left(p_{2 k+2}\right)$ for $1 \leq k \leq g$. We now need to compute the vector $R$, i.e to compute the vector of Riemann constants and subtract from it the sum of $A$ 's. The result is certainly classical: $R=Q=A\left(p_{2}\right)$. To prove this one can note that by definition $R$ is the unique vector such that $\theta(A(p)+R)$ as a function of $p \in C$ has precisely $g$ zeroes at $A^{-1}\left(A_{k}\right)$, i.e. at $p_{2 k+2}$. To check that this is the case we note that $A\left(p_{2}\right)+A\left(p_{2 i}\right)$ is always odd, as a theta characteristic, so that even Riemann's theta function will vanish at the point $A\left(p_{2}\right)+A\left(p_{2 i}\right)$; thus $R=A\left(p_{2}\right)$.

Let us now substitute all this into formula (8). We have $\alpha=\alpha_{0}=0, \beta=\beta_{0}=e_{1}$, $\alpha_{k}=s_{k}$ and $\beta_{k}=e_{k+1}$, where we understand $e_{g+1}$ to be zero. Since all theta functions of the second order are periodic with respect to $z \rightarrow z+e_{i}$, the additional integer shifts by $s_{i}$ do not matter, and finally (8) yields

THEOREM 10. An irreducible period matrix $\tau \in \mathcal{H}_{g}$ is the period matrix of a hyperelliptic Jacobian with the basis of cycles chosen as above if and only if the following cubic identity for theta functions of the second order is satisfied for all $\sigma \in(\mathbb{Z} / 2 \mathbb{Z})^{g}$ and for all $z \in X_{\tau}$ (and thus for all $z \in \mathbb{C}^{g}$ ):

$$
\begin{aligned}
& \sum_{\varepsilon} \Theta[\varepsilon](z) \Theta[\varepsilon](z) \Theta[\sigma](z) \\
= & \sum_{\varepsilon} \sum_{k=0}^{g}(-1)^{\left(\varepsilon+\sigma, e_{k+1}\right)} \Theta[\varepsilon](z) \Theta\left[\varepsilon+s_{k}\right] \Theta\left[\sigma+s_{k}\right](z),
\end{aligned}
$$

where we understand $e_{g+1}$ to be zero.

To check that this makes sense let us do the computations in low genus and see what we get. To simplify formulas, we write $[\varepsilon]$ for $\Theta[\varepsilon](z)$. We order the $\varepsilon$ for summation of the terms of (9) lexicographically to keep track of where we are.

Genus 2: We do not expect to get any meaningful equations, as any irreducible abelian variety of dimension two is a hyperelliptic Jacobian, so our characterization should be vacuous. We verify this; here is what formula (9) yields for $\sigma=00$ :

$$
\begin{array}{ll} 
& {[00][00][00]+[01][01][00]+[10][10][00]+[11][11][00]} \\
= & {[00][00][00]+[01][01][00]-[10][10][00]-[11][11][00]} \\
+ & {[00][10][10]-[01][11][10]+[10][00][10]-[11][01][10]} \\
+ & {[00][11][11]+[01][10][11]+[10][01][11]+[11][00][11]}
\end{array}
$$

and all the terms cancel. A similar computation shows that the identity is also trivial for all other choices of $\sigma$.

Genus 3: Here we have $\operatorname{dim} \mathcal{J}_{3}=\operatorname{dim} \mathcal{A}_{3}=6$, while $\operatorname{dim} \mathcal{I}_{3}=5$, so we should have a non-trivial identity. Indeed let us choose $\sigma=000$ and write down (9) in this case; after multiple cancellations and dividing by two it becomes simply

$$
\begin{aligned}
& \Theta[000](z) \Theta[101](z) \Theta[101](z)+\Theta[011](z) \Theta[101](z) \Theta[110](z) \\
= & \Theta[010](z) \Theta[101](z) \Theta[111](z)+\Theta[001](z) \Theta[100](z) \Theta[101](z),
\end{aligned}
$$


which using formula (3) is equivalent to

$$
\Theta[101](z) \cdot \theta\left[\begin{array}{l}
101 \\
111
\end{array}\right](2 z) \cdot \theta\left[\begin{array}{l}
101 \\
111
\end{array}\right](0)=0 .
$$

Choosing a different $\sigma$ yields a different equation: in general we get

$$
\Theta[101+\sigma](z) \cdot \theta\left[\begin{array}{c}
101 \\
111
\end{array}\right](2 z) \cdot \theta\left[\begin{array}{l}
101 \\
111
\end{array}\right](0)=0
$$

All of these equations together are equivalent to $\theta\left[\begin{array}{l}101 \\ 111\end{array}\right](0)=0$, since theta functions of the second order never all vanish simultaneously and $\theta\left[\begin{array}{l}101 \\ 111\end{array}\right](2 z)$ cannot be identically zero in $z$. Now to actually characterize $\mathcal{I}_{3} \subset \mathcal{A}_{3}$ we need to get rid of the condition "that the basis of cycles is chosen as above" in theorem 10. But choosing a different basis of cycles means acting on the period matrix by a symplectic transformation. Since theta constants are modular with respect to $\Gamma(2,4)$, conjugating equation (10) by any $\gamma \in \Gamma(2,4)$ would not change it, so we only need to act by the finite group $\operatorname{Sp}(2 g, \mathbb{Z}) / \Gamma(2,4)$. It is well known that the action of this group is transitive on the set of even theta characteristics (see $[\mathrm{I}]$ ), so we can get the vanishing of a theta constant with any even characteristic.

Proposition 11. An irreducible abelian variety of genus 3 is a hyperelliptic Jacobian if and only if it has a vanishing theta constant with even characteristic. This is known classically, see [M].

Genus 4: here the situation is more interesting: Mumford's conditions include some non-vanishing, so getting the explicit equations for the closure of $\operatorname{Th}\left(\mathcal{I}_{4}\right) \subset T h\left(\mathcal{A}_{4}\right)$, without any inequalities serving to cut off the extra components inside the reducible locus, would be interesting. For the case of $\sigma=0000$ the equation we get from (9) after cancellations becomes the following cubic (we have rearranged the terms lexicographically and omitted square brackets):

$$
\begin{aligned}
& 0000 \cdot 1001 \cdot 1001+0000 \cdot 1010 \cdot 1010+0000 \cdot 1011 \cdot 1011+0000 \cdot 1101 \cdot 1101 \\
& 0011 \cdot 1101 \cdot 1110+0101 \cdot 1000 \cdot 1101+0101 \cdot 1011 \cdot 1110+0110 \cdot 1010 \cdot 1100 \\
& 0111 \cdot 1001 \cdot 1110+0111 \cdot 1011 \cdot 1100=0001 \cdot 1000 \cdot 1001+0001 \cdot 1100 \cdot 1101 \\
& 0010 \cdot 1000 \cdot 1010+0010 \cdot 1101 \cdot 1111+0011 \cdot 1000 \cdot 1011+0100 \cdot 1010 \cdot 1110 \\
& 0100 \cdot 1011 \cdot 1111+0101 \cdot 1001 \cdot 1100+0101 \cdot 1010 \cdot 1111+0110 \cdot 1001 \cdot 1111
\end{aligned}
$$

while for example for $\sigma=0001$ we get

$$
\begin{aligned}
& 0000 \cdot 1000 \cdot 1001+0000 \cdot 1100 \cdot 1101+0010 \cdot 1001 \cdot 1010+0011 \cdot 1001 \cdot 1011 \\
& 0011 \cdot 1100 \cdot 1110+0100 \cdot 1000 \cdot 1101+0100 \cdot 1011 \cdot 1110+0101 \cdot 1010 \cdot 1110 \\
& 0101 \cdot 1011 \cdot 1111+0111 \cdot 1000 \cdot 1110=0001 \cdot 1000 \cdot 1000+0001 \cdot 1010 \cdot 1010 \\
& 0001 \cdot 1011 \cdot 1011+0001 \cdot 1100 \cdot 1100+0010 \cdot 1100 \cdot 1111+0100 \cdot 1001 \cdot 1100 \\
& 0100 \cdot 1010 \cdot 1111+0110 \cdot 1000 \cdot 1111+0110 \cdot 1010 \cdot 1101+0111 \cdot 1011 \cdot 1101
\end{aligned}
$$

Neither of these cubics is equal to $\Theta[\delta](z) \theta\left[\begin{array}{l}\alpha \\ \beta\end{array}\right](2 z) \theta\left[\begin{array}{l}\alpha \\ \beta\end{array}\right](0)$ for any $\alpha, \beta, \delta$. However, from theorem 10 we see that

Proposition 12. The vanishing of the full set of 16 cubics similar to the ones above, for all $\sigma$, identically in $z$ characterizes a component of $T h\left(\mathcal{I}_{4}(2,4)\right) \subset$ $\operatorname{Th}\left(\mathcal{A}_{4}^{\text {irr }}(2,4)\right)$. 
By Mumford's and Poor's results such a component is also determined by identical vanishing of some set of theta constants with characteristics. Thus the vanishing of our 16 cubics should imply, for irreducible abelian varieties, the vanishing of some theta constants with characteristics and vice versa, but we are now unable to see this directly.

The difficulty in doing so is not only due to the fact that the cubic equations are very complicated. Indeed, thinking of each cubic $f_{\sigma}$, evaluated at $z=0$, as a polynomial on $\mathbb{P}^{15}$ with zero locus $Z\left(f_{\sigma}\right)$, we can only say that $\underset{\sigma}{\cap} Z\left(f_{\sigma}\right) \cap T h\left(\mathcal{A}_{4}^{\text {irr }}(2,4)\right)$ is contained in the common zero locus of some quadrics (which are by (3) the expressions for theta constants with characteristics in terms of theta constants of the second order) on $T h\left(\mathcal{A}_{4}^{i r r}(2,4)\right)$. It may in fact not be the case that the whole $\cap Z\left(f_{\sigma}\right)$ is contained in the zero locus of these quadrics in $\mathbb{P}^{15}$. Thus to be able to see the relation of the vanishing of our cubics to the vanishing of theta constants with characteristics, we may need to know the equations for the closure of $T h\left(\mathcal{A}_{4}^{i r r}(2,4)\right) \subset \mathbb{P}^{15}$, which are not known.

The above discussion was for just one component of $T h\left(\mathcal{I}_{4}(2,4)\right)$. The equations for the other components corresponding to different choices of the basis of cycles on the curve are of course obtained by acting on the set of 16 cubics by elements of $\operatorname{Sp}(8, \mathbb{Z}) / \Gamma(2,4)$.

FinAL REMARK. It seems likely that in any genus evaluating equations (9) only at $z=0$ for all $\sigma$ should yield the defining set of equations for a component of $T h\left(\mathcal{I}_{g}(2,4)\right)$. Indeed using $(7)$ it can be easily shown that if these are satisfied, then (9) is satisfied for $z$ being any point of order two. Thus both sides of (9) are sections of $6 \Theta$ that agree at all points of order two, and one would hope that then they agree everywhere and give the same function of $z$, so that (9) is true identically and we can apply theorem 10 .

Acknowledgements. We would like to thank Professor Igor Krichever for bringing to our attention and explaining to us the works [BK1] and [BK2] and the conjectural characterization of Jacobians by the addition formula, which got this work started, Professor Robert Gunning for helpful discussions on addition formulas for theta functions and multisecants, and Professor Emma Previato for valuable discussions of the equations for Kummer and modular varieties.

I owe a debt of gratitude to Professor Yum-Tong Siu for teaching me and sharing with me his insights and ideas in moduli theory.

It is an honor for me to dedicate this paper to Professor Yum-Tong Siu on the occasion of his 60 th birthday.

\section{REFERENCES}

[BK1] Buchstaber, V.M., Krichever, I.M., Vector addition theorems and Baker-Akhiezer functions, Teor. i Mat. Fizika, 94:2 (1993), pp. 200-212.

[BK2] Buchstaber, V.M., Krichever, I.M., Multidimensional vector addition theorems and the Riemann theta functions, Internat. Math. Res. Notices, 10 (1996), pp. 505-513.

[I] IGusa, J.-I., Theta functions. Die Grundlehren der mathematischen Wissenschaften, Band 194. Springer-Verlag, New York-Heidelberg, 1972.

[F] FARKAS, H., Schottky-Jung theory, Theta functions - Bowdoin 1987, pp. 459-483.

[Gr] Grushevsky, S., Effective Schottky problem, Ph.D. Dissertation, Harvard University, 2002.

[Gu1] Gunning, R. C., Some curves in abelian varieties, Invent. Math., 66:3 (1982), pp. $377-389$.

[Gu2] Gunning, R. C., Some identities for abelian integrals, Amer. J. Math., 108:1 (1986), pp. $39-74$. 
[M] Mumford, D., Tata lectures on Theta II, Progress in Math. vol. 43, Birkhäuser, Boston, 1984.

[P] Poor, C., The hyperelliptic locus, Duke Math. J., 76:3 (1994), pp. 809-884.

[SM] Salvati Manni, R., Modular forms vanishing on the hyperelliptic locus, Japan. J. Math. 29:1 (2003), pp. 135-142.

[S] Schотткy, F., Zur Theorie der Abelschen Functionen vor vier Variablen, J. Reine Angew. Math., 102 (1888), pp. 304-352.

[T] Thomae, J., Beitrag zur Bestimmung von $\theta(0,0, \ldots, 0)$ durch die Klassenmoduln algebraischer Funktionen, J. Reine Angew. Math., 71 (1870), pp. 201-222.

[We] Welters, G., A criterion for Jacobi varieties, Ann. of Math. (2), 120:3 (1984), pp. $497-504$.

[Wi] Wirtinger, W., Untersuchungen über Thetafunktionen, Leipzig: Teubner 1895. 